\title{
A Battery Health Data Sharing Model via Blockchain
}

\author{
Peng Ding \\ College of Mechanical and Automotive Engineering, Shanghai University of Engineering Science, Shanghai, China \\ Email: cumtdp0122@163.com
}

How to cite this paper: Ding, P. (2018) A Battery Health Data Sharing Model via Blockchain. Open Access Library Journal, 5: e5016.

https://doi.org/10.4236/oalib.1105016

Received: October 30, 2018

Accepted: November 16, 2018

Published: November 19, 2018

Copyright (c) 2018 by author and Open Access Library Inc.

This work is licensed under the Creative Commons Attribution International License (CC BY 4.0).

http://creativecommons.org/licenses/by/4.0/

(c) (7) Open Access

\begin{abstract}
According to the status quo of the electrical vehicle market, the numbers of retired power battery from the NEV (new electrical vehicle) would definitely lead to a big increase. And the key factor to evaluate the $\mathrm{SOH}$ (state of health) of the retired battery is to get the historical data to optimize the parameters of the models. But the truth is there are many restrictions in data access and sharing between the battery manufacturers, EVH manufacturers and the users of these retired battery. To solve this problem, we propose a blockchain-based battery data sharing model, with advantage of anti-fake, digital signature, easy to trace back, as well as decentralization, collective maintenance and tamper resistance. We put this system in the use of life prediction and rating evaluation of the retired battery and identify the responsible for battery accidents. In addition, we simulate the different part of the battery industrial chain with three Raspberry-pi and use our owe blockchain platform to run this application. Finally, the merits and impacts of this model are presented and analyzed by comparisons in terms of existing battery central data base.
\end{abstract}

\section{Subject Areas}

Mechanical Engineering

\section{Keywords}

Battery Data, Protocol Transform, Blockchain, Sharing Model

\section{Introduction}

A large number of batteries from NEV start to reach the guarantee time and wait for recycling. But the series problem now is when you face a bunch of the retired battery, how could you judge if these battery are good enough for the next jobs? 
Though the batteries actually generate thousands of working data per day, the truth is that these data stored in different database which are totally isolated and barely interact with each other. How to connect these data and guarantee the authenticity of these data is a realistic topic.

This paper studies the data collection from the all kinds of sensor, data analysis from the analysis database, report generation from the battery information, and using MD5 to generate the abstract of the report and transfer it to the blockchain network for persistent recording, and the information stored in the blockchain system cannot be tampered. Finally, it is convenient for different parts to apply for access to relevant data and trace back the relevant responsibilities when the battery hit troubles. By setting up a reasonable incentive mechanism, the whole system can operate stably and independently, so that each part in the whole battery manufactory chain can focus more on the improvement and development of their own business, and at the same time, promote the stable and efficient operation of the whole system.

\section{Test SOH of Lithium Ion Power Batter}

\subsection{Battery Healthy State Estimation}

As we all know, after a long time work, the power battery system will continue to decay to the retired edge, so how to effectively evaluate the battery health is not only offer the important basis parameters for the calculation of SOC (state of charge), SOP (state of power) but at the same time to judge whether power battery system need to be changed. At the same time, different application scenarios have different requirements on the battery, and there are multiple parameters could affect the process of the attenuation (SOC interval, multiple rates, environmental temperature, etc.) (Figure 1) For example, EV models usually open the SOC interval of $20 \%$ to $100 \%$, while PHEV models tend to open the SOC interval of a little bit, once they enter the low SOC interval, they will enter the CS mode [1].

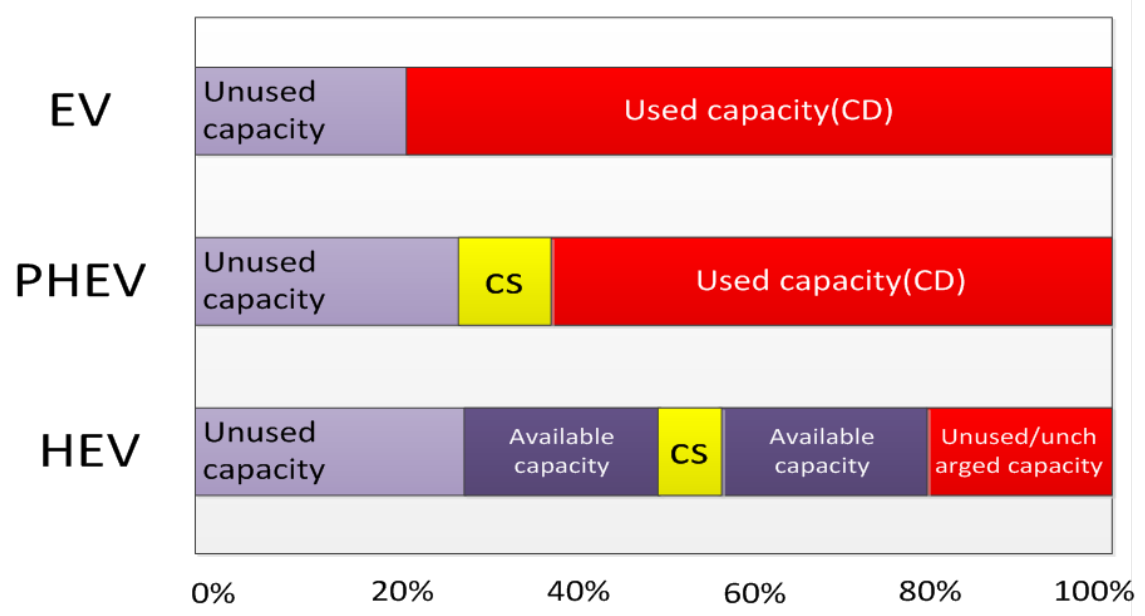

Figure 1. Different requirements of the power battery. 
Therefore, in the process of the evaluation, it is necessary to evaluate the battery life according to the actual operating conditions, so that the battery system can meet the application requirements of the whole life cycle as much as possible, and only the test experiment design combined with the actual operating conditions can make the subsequent $\mathrm{SOH}$ estimation feasible.

However, the attenuation process of lithium battery is a typical nonlinear electrochemical system, and the chemical internal characteristic parameters are not easy to be measured, which lead to evaluate $\mathrm{SOH}$ inaccurately. So we should find another kind of the data which are easy to get to evaluate the SOH. Multiple researchers have found out that the estimation of $\mathrm{SOH}$ is usually analyzed from two different dimensions. The first dimension is based on the electrochemical mechanism, and the observation content is dominated by internal reaction mechanisms such as loss of active lithium ions and collapse of material lattice. The second dimension is the dimension based on the performance of battery external characteristics, which is dominated by the observation of external characteristic parameters such as current, voltage and temperature. Because the internal characteristics involved in the former are not easy to be measured, the BMS algorithm usually uses the second dimension to evaluate $\mathrm{SOH}$ [2]. This paper also mainly discusses the $\mathrm{SOH}$ estimation method based on external characteristics (Figure 2).

As consumers, our experience with batteries tends to be that the longer we use them, the worse battery power performance it would be. In general, power battery performance will continue to "degrade" along with "operation". At the algorithm level, "run use" is generally broken down into calendar life and cycle life, while "performance fade" is broken down into "Capacity fade" and "DCR growth" [3]. Through a series of core tests, we try to find the relationship between the above factors.

\subsection{Battery Attenuation Experiment}

Figure 3 shows the schematic diagram of the whole experiment, we use the 80 $\mathrm{v} / 60 \mathrm{Ah}$ battery pack as the measured object.

We use Repower to charge and discharge the battery pack. All the experiment run under the $24^{\circ} \mathrm{C}$ environment. We set different operation conditions to check out how those factor effect the health of battery.

\subsection{Result Analysis of Battery Attenuation}

After long-term test and experiments, it is generally believed that the charge-discharge ratio (C rate), discharge depth (DOD), and temperature ( $\mathrm{T}$ ) play a key role in the attenuation of battery performance. Therefore, current, monomer voltage, SOC, temperature and scheduling time are the most important input parameters in the cycle life model (Table 1).

The first experiment to check out the how temperature influence the attenuation of the battery. We put the battery in three different temperature $0^{\circ} \mathrm{C}, 30^{\circ} \mathrm{C}$, 


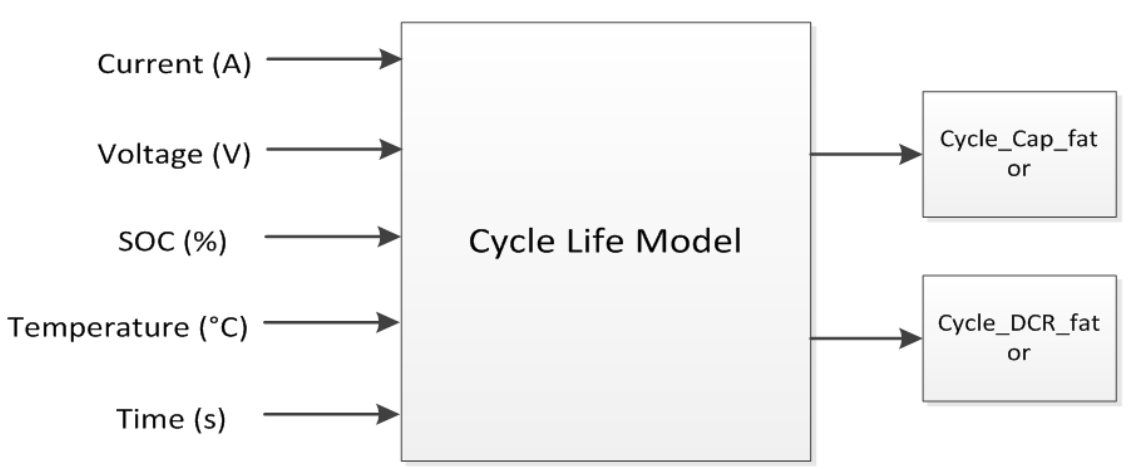

Figure 2. The basic external characteristics of battery model used.

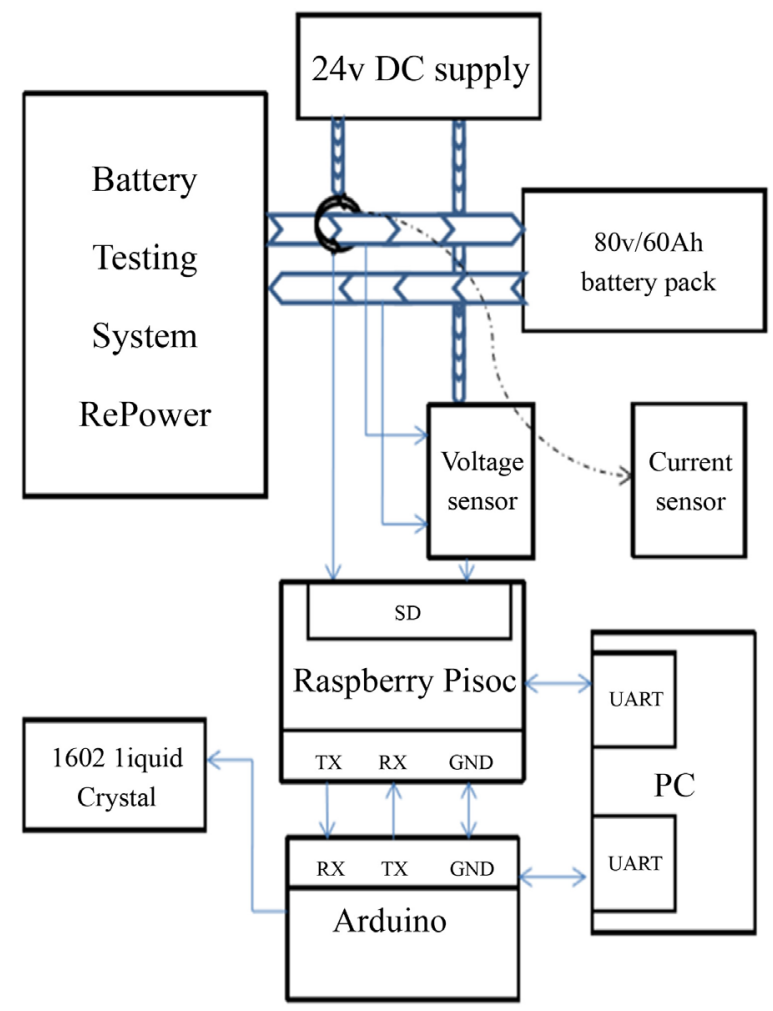

Figure 3. Battery data collection system.

Table 1. How temperature affect the attenuation process.

\begin{tabular}{ccccc}
\hline \multirow{2}{*}{ Attenuation Test } & \multicolumn{4}{c}{ Step1. How DOD Effect the Attenuation Process } \\
\hline & Number & DOD $(\%)$ & $\mathrm{T}\left({ }^{\circ} \mathrm{C}\right)$ & C Rate \\
\hline 1 & 170 & 0 & 1 \\
3 & 70 & 30 & 1 \\
& 70 & 60 & 1 \\
\hline
\end{tabular}

$60^{\circ} \mathrm{C}$ respectively, and keep putting test battery in the cycle of charge, impedance and discharge until the battery decline by $30 \%$ from the original capacity. Figure 4 shows the results of the experiment. 


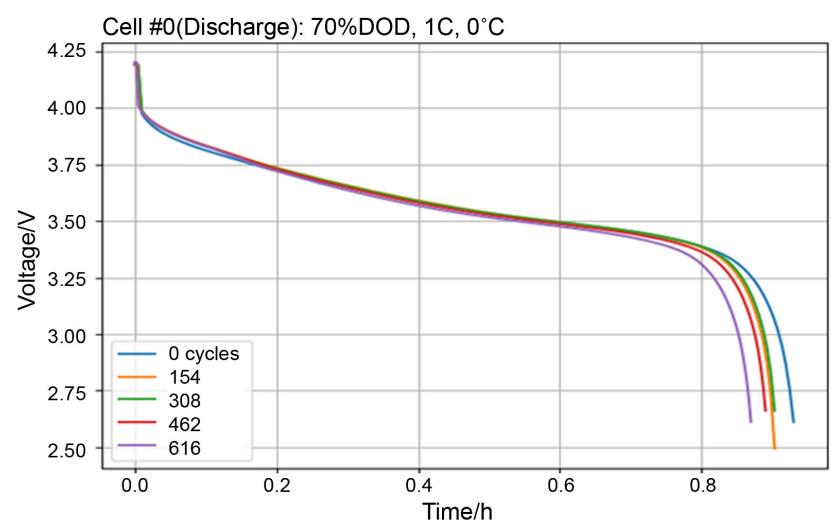

(a)

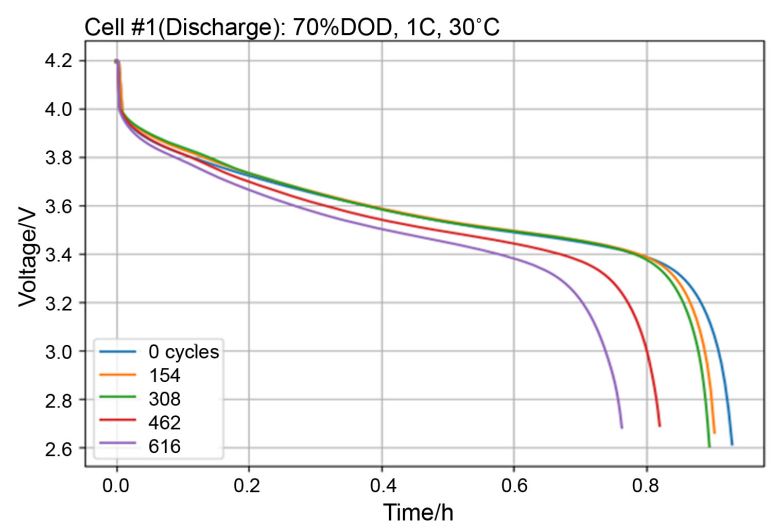

(b)

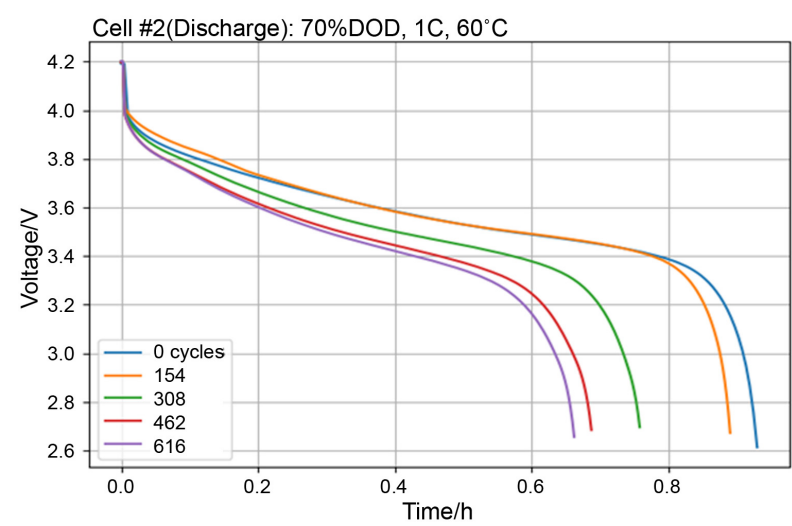

(c)

Figure 4. Battery accelerated aging test under different temperature. (a) $70 \% \mathrm{DOD}, 1 \mathrm{C}, 0^{\circ} \mathrm{C}$; (b) $70 \% \mathrm{DOD}, 1 \mathrm{C}, 30^{\circ} \mathrm{C}$; (c) $70 \% \mathrm{DOD}, 1 \mathrm{C}, 60^{\circ} \mathrm{C}$.

Analysis results show that under the same number of charge and discharge cycle, as the temperature rise, the batteries are more easily to get old. So we should pay special attention to the temperature factor when we evaluate the battery life decline (Table 2).

Table 2 is aimed to study on how DOD (depth of discharge) influences the process of the battery capacity decline. The average situation of the discharge is from $4.2 \mathrm{v}$ to $2.7 \mathrm{v}$, for the purpose to get the different DOD, we should set the different stop voltage while discharging. We decide to set 5 layers, which are 3.45 $\mathrm{v}, 3.2 \mathrm{v}, 3.05 \mathrm{v}, 2.90 \mathrm{v}$, and $2.85 \mathrm{v}$ separately, which are corresponding DOD are $50 \%, 60 \%, 70 \%, 80 \%$ and $90 \%$. Through this experiment we could find out compared to temperature, if the battery capacity decline is more sensitive to the DOD.

Left result of Figure 5 shows the relation between battery capacity attenuation with cycles in different DOD. To more easy to compare, transfer the cycles to time. Through the experiment we could find that compared to the temperature, the battery capacity attenuation is lower sensitive to DOD.

\section{Data Persistence with Blockchain}

Blockchain technology is not only one technology, but the integration of multiple 
Table 2. How DOD affect the attenuation process.

\begin{tabular}{ccccc}
\hline \multirow{2}{*}{ Attenuation Test } & \multicolumn{4}{c}{ Step2. How DOD Effect the Attenuation Process } \\
\cline { 2 - 5 } & Number & DOD $(\%)$ & T $\left({ }^{\circ} \mathrm{C}\right)$ & C Rate \\
\hline 2 & 50 & 25 & 1 \\
3 & 60 & 25 & 1 \\
4 & 70 & 25 & 1 \\
5 & 80 & 25 & 1 \\
\hline
\end{tabular}

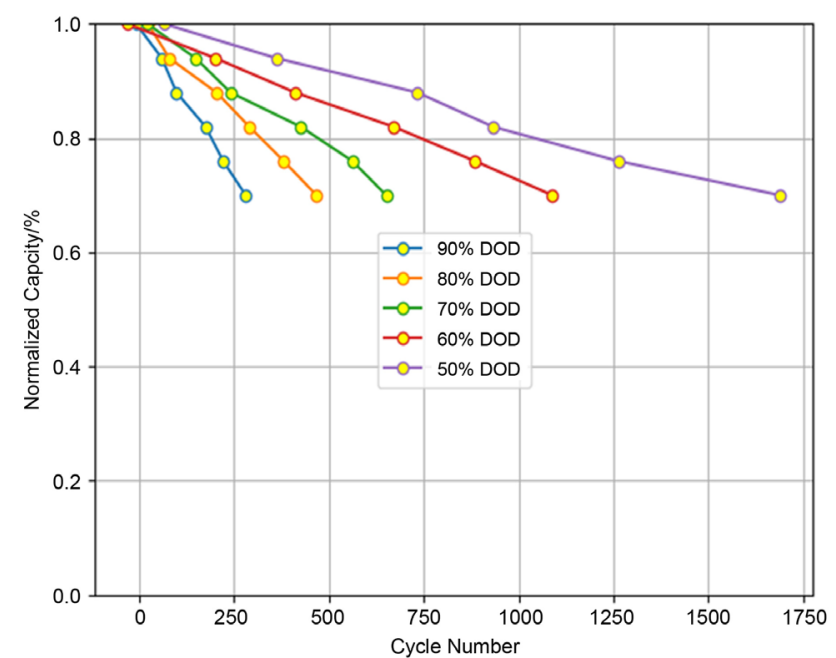

(a)

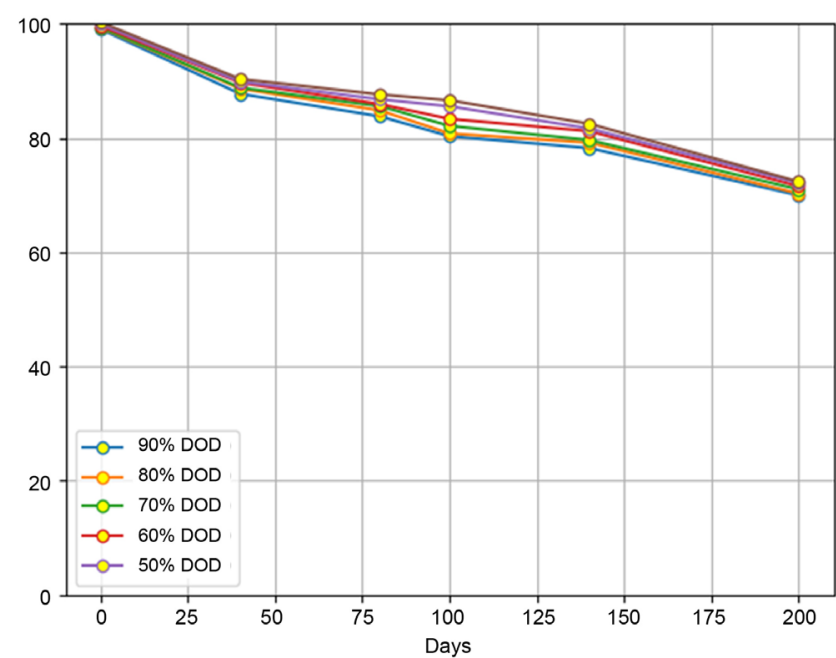

(b)

Figure 5. Battery accelerated aging test under different DOD. (a) Capacity decline under number; (b) Capacity decline under date.

technologies. As shown in Figure 6, the block chain can be divided into six layers compared with OSI protocol [4] [5], which are data layer, network layer, consensus layer, incentive layer, contract layer and application layer.

\subsection{Data Layer}

The structure of blockchain contains the head and body, head store the special information include the Merkel root, the timestamp and nonce and difficult factor which generated by mining in Bitcoin. The most import information in the head is the hash number of self-block and the hash number of last-block, which make the all the blocks connect together like Figure 7 shows. The body records every single transaction data.

Rather than motivating by bookkeeping, we design the motivation and punishment by the battery health data. And in the block body we stored the every single battery heath data.

\subsection{Network Layer}

After all, blockchain technology runs on the Internet. Compared with the openness of Internet interaction, blockchain actually adds many rules to guarantee 


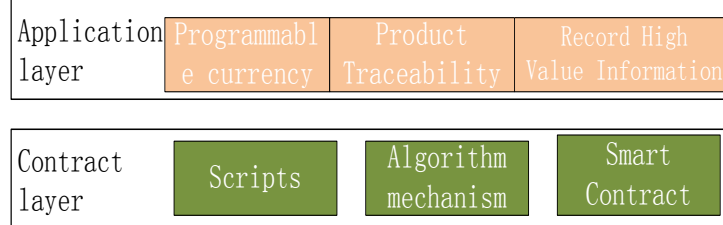

\begin{tabular}{|l|l|}
\hline $\begin{array}{l}\text { Incentive } \\
\text { layer }\end{array}$ & $\begin{array}{l}\text { Distribution } \\
\text { mechanismism }\end{array}$ \\
\hline
\end{tabular}

\begin{tabular}{|c|c|c|c|c|c|}
\hline Consensus & POW & PoS & DPoS & PBFT & $\cdots$ \\
\hline
\end{tabular}

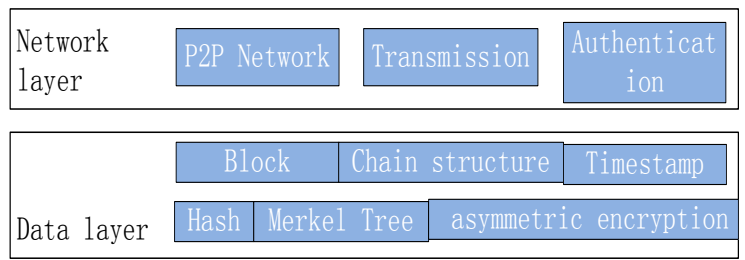

Figure 6. Six layers of blockchain.

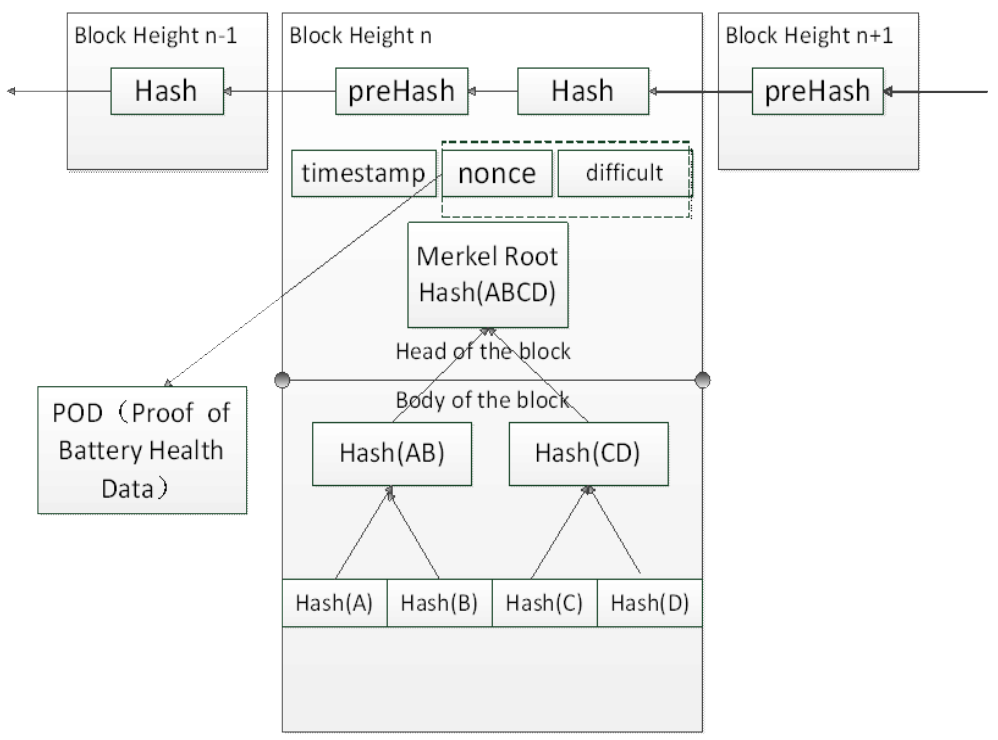

Figure 7. The structure of blockchain.

the high coupling and tampering of valuable information. The network layer of blockchain contains P2P network, network transmission and network verification. For the network communication layer, the first thing to be recorded is valuable information sorted by time or by remuneration, which is also the information recorded by blockchain as a Shared ledger.

Though the network layer could guarantee all the information should be checked before record on blockchain, but considering the huge amount of the battery data, it's not realistic to record all of the battery data. So we design the database architecture. Whether the power battery used for EV or building storage, the data should be transfer to the local database and generate a battery 
health state report termly and automatically based on agreement. The digest of these reports will be sent to the blockchain network, like Figure 8 shows. After applying for the access of these documents and getting the permission, the applicant could compare the documents with the digest stored in blockchain to check out if these information are the original one and have not be changed

\subsection{Consensus Layer and Incentive Layer}

These two layer always be put together is because to reach the consensus there must be the incentive or punishment. In bitcoin world, they use the POW (proof of work) consensus to reach the agreement, which means if someone did more jobs to push the whole book-keeping forward than he will get the reward. The amazing thing about the bitcoin is that each part of the bitcoin society member does the jobs for their owe benefits, than the whole bitcoin system stably works for ten years with no one claimed responsible for the bitcoin. So we can see that a well-designed consensus and incentive mechanism will promote the blockchain system functional efficiently and automatically. But as we all know the POW will cause a large amount of electricity waste. Using the blockchain to store the battery data is not necessary to use the public blockchain as the bitcoin or ethereum system. We could use Hyper-Ledger Fabric as a reference, which uses the quality of the battery data to offer the award or punishment.

\subsection{Contract Layer}

The symbol of blockchain 2.0 is the birth of the blockchain platform with the ability to write smart contract like ethereum. Smart contracts are programmatic environments with Turing complete logic [6] based on the blockchain network. Smart contract is aimed to perform the contract automatically based on certain triggering mechanism. In our batter health data sharing blockchain platform, a very useful application scenario is when members of this consortium proposed a new type of consensus mechanism, once the proposal agreed by certain number of the members, it will pass and deploy to the whole system automatically.

\section{Identify Verification and Accessibility}

\subsection{Asymmetric Encryption}

Data confidentiality is involved in many application scenarios of consortium chain, and many blockchain solutions are implemented through asymmetric encryption technology. The main application scenarios of asymmetric encryption are data encryption and digital signature. The advantage of asymmetric encryption over symmetric encryption is that it does not need to synchronize the decryption information, which avoids the interception of the key during transmission.

Asymmetric encryption is implemented by having two keys-public key and private key. Public key broadcast in the whole internet, everyone can get the public key information, but only the sender has the private key (Figure 9). So 


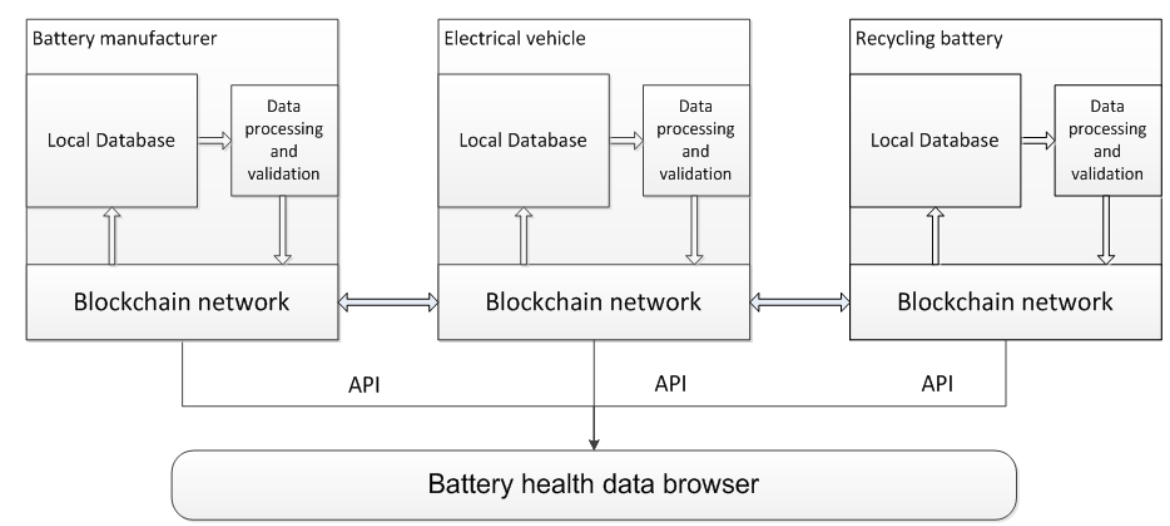

Figure 8. The database architecture.

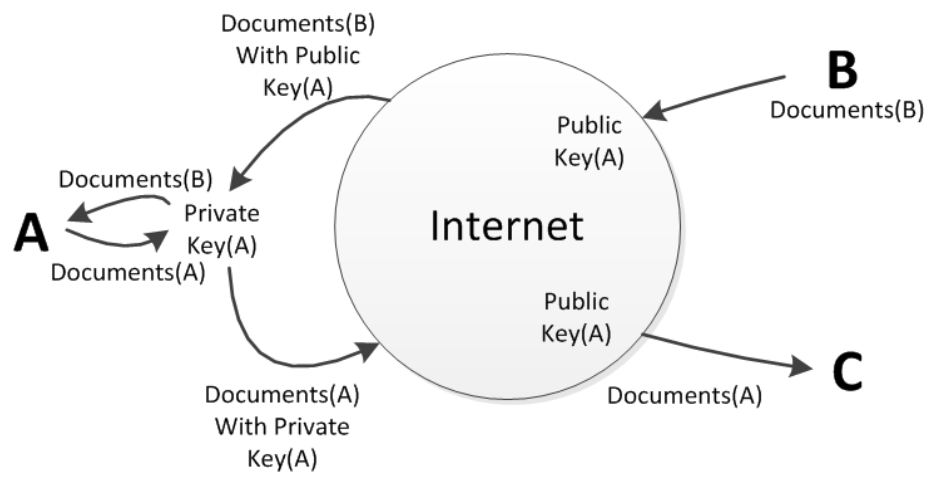

Figure 9. Asymmetric encryption schematic diagram.

that all the people in the internet can use A's public key to encrypt files, but only A can get the information to use the private key to decrypt the file. Or A encrypts the file by its private key. Any node in the network can use A's public key and get the file information. At this point, it can be proved that the owner of the file is indeed A. This is the application scenario of asymmetric encryption used in encryption, decryption and digital signatures. Two typical application scenarios for asymmetric encryption are described below.

\subsection{Message Digest}

When applying for the battery sensitive information, we use asymmetric encryption as our signature certification. Assuming the battery manufacturer $A$ signs the battery health report with its own private key and then transfer to the blockchain network, once this report is downloaded and decrypted by $A$ 's public key. The truth of this report is totally guaranteed by $A$.

This model adopts RSA asymmetric encryption and adopts the lattice algorithm in [7] to design the protocol.

1) Generate the random matrix $A \in Z_{q}^{n^{*} n}$, chose the safe integer $q, n$

2) Generate public or private key: public key $p k=P, P=R-A, S \in Z_{q}^{n^{* 1}}$, $R, S$ are the Gauss parameters, private key is $s k=S$

3) Encryption algorithm: 


$$
c=\left(c_{1}, c_{2}\right)=\left(e_{1} A+e_{2}, e_{1} P+e_{3}+m\left|\frac{q}{2}\right|\right)
$$

$e_{1}, e_{2}, e_{3}$ are the error parameters.

4) Decryption algorithm:

$$
m=c_{1} S+c_{2}
$$

Determine 0 or 1 based on the distance from 0

5) Re-encryption algorithm to generate the private key:

$$
\begin{aligned}
& r k_{a \rightarrow b}=\left(P_{B}, Q\right) \\
& Q=\left[\begin{array}{cc}
X & -X S_{B}+E+S_{A} \\
0 & I
\end{array}\right]
\end{aligned}
$$

$X$ is the random matrix, $E$ is the noise.

6) Re-encryption algorithm:

$$
\left(c_{1}^{\prime}, c_{2}^{\prime}\right)=h_{1}\left(A, P_{B}\right)+\left(h_{2}, h_{3}\right)+\left(c_{1}, c_{2}\right) Q
$$

$h_{1}, h_{2}, h_{3}$ are the error parameters

Figure 10 shows the three steps the battery health information be recorded on the blockchain. First step, generate the report in the local database termly and automatically based on agreement. Step two, using MD5 (message digest 5) [8] toolkit to generate the digest. Sept three, put the digest to the blockchain system.

\subsection{Accessibility and Verification}

In our system, the valued information stored in the blockchain network is just the message digest, which is not what we want. Figure 11 shows the process of the accessibility and verification when applying for battery data stored in different local database.

Battery Test Report

$$
\text { Test Date: } 0 \mathrm{ct}_{2} 29,2018
$$

1. Battery charge/discharge experiment

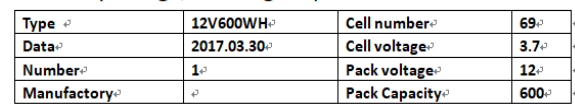

2. Using PWM to charge the battery till fully charged

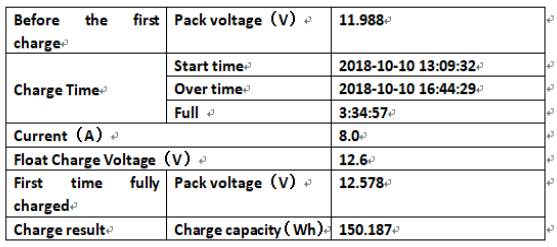

Charge result $\quad$ Charge capacity $($ Wh $)=150.187$

3. Battery discharge for 10 hours

\begin{tabular}{|l|l|l|}
\hline \multicolumn{2}{|l|}{ Discharge current $(\mathrm{A})$} & $\mathbf{8 . 0}$ \\
\hline \multirow{3}{*}{ Discharge time } & Start time & $\mathbf{2 0 1 8 - 1 0 - 1 0 1 6 : 4 9 : 4 5}$ \\
\cline { 2 - 3 } & Over time & $\mathbf{2 0 1 8 - 1 0 - 1 0} 22: 33: 09$ \\
\cline { 2 - 3 } & Discharge time & $\mathbf{5 : 4 3 : 2 5}$ \\
\hline Pack voltage & Startvoltage $(\mathrm{V})$ & 12.572 \\
\cline { 2 - 3 } & & \\
\hline
\end{tabular}

Figure 10. Recording on blockchain with report digest.
Report Digest

-BEGIN RSA PUBLIC KEY-----

AoGBAIZ99S4EXCOR7S7BKos0XI3luWeTVHy/xhRgkVRSsbsLg3tYa3/P+u2K 6fh+wy5kRxiqwGIuaGddX24v7NMr/xIhy9yeldi6kIj39piI2wrPotTnJ0Cp Pi3c0cZBaMVJm50EBk0sB/dxfSLqwr j331BpAUwUpYVLpPgFAgMBAAE= -END RSA PUBLIC KEY-----

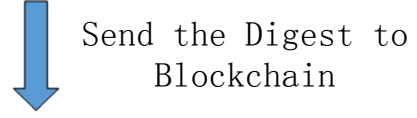

Welcome to login to your account address:

ba0e9b343c1b47c6a3de42aa17725957

Block Message:

['chain': ["'index': 1, 'timestamp': 1533350520, 'transactions': ['the great day to start to bulld a totally new energy system'], 'proof: 10

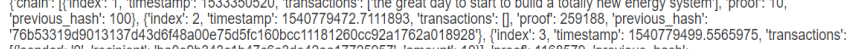

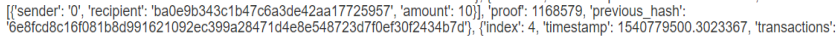

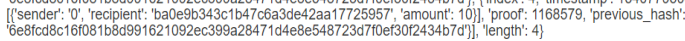

Current block height: 4 


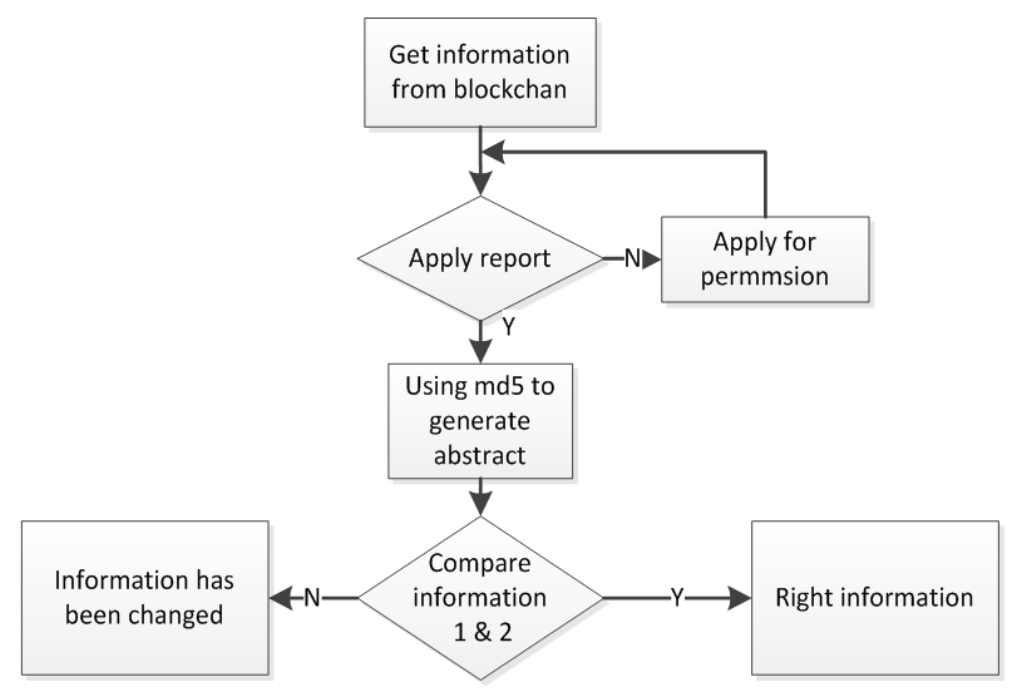

Figure 11. Process of the accessibility and verification.

The blockchain network here guaranteed the battery data required from other database is really the original one. Once the data changed, the verification process will detect and alert.

\section{Conclusions}

Battery capacity will keep declining along with working. If we could use the battery data generated in the daily working, we could evaluate the batter health condition accurately. But how could we get access to the data? How could we guarantee whether the data are safe and not be changed? We try to create a system to solve this problem.

First we do the experiment to put the battery in all kinds of different situation to find out how the environment factors work on the battery attenuation. The data shows that the temperature has more effects on the battery attenuation than DOD and $\mathrm{C}$ rate. So when we design the report we should pay more attention on the temperature factor than others.

Then we design the blockchain network which is more like a consortium blockchain. This blockchain offers a reliable channel for members in the battery data sharing chain platform. All the members should upload the battery data on the blockchain with their signature, and the regulation of the data is made by more than half of the members. After get the permission and download the data from other database, the blockchain network will verify the whether the report is fake one. With the appropriate Consensus and incentive mechanism, the whole system will work stably and automatically.

This article proposes a combination of IOT (internet of things) and blockchain network. The battery data are stored in our blockchian system. Compared to the central database, the distributed database with blockchain technology will make the information more open and transparent and create a trustful environment to transfer data. 


\section{Acknowledgements}

First and foremost, I would like to show my deepest gratitude to my supervisor, Dr. Yan Xiao, a respectable, responsible and resourceful scholar, who has provided me with valuable guidance in every stage of the writing of this thesis. Without his enlightening instruction, impressive kindness and patience, Icould not have completed my thesis. His keen and vigorous academic observation enlightens me not only in this thesis but also in my future study. I would also like to thank all my teachers who have helped me to develop the fundamental and essential academic competence. My sincere appreciation also goes to the teachers and students from Shanghai University of Engineering and Technology who participated this study with great cooperation. Last but not least, I would like to thank all my friends, especially my three lovely roommates, for their encouragement and support.

\section{Conflicts of Interest}

The author declares no conflicts of interest regarding the publication of this paper.

\section{References}

[1] Tsujikawa, T., Yabuta, K., Arakawa, M. and Hayashi, K. (2013) Safety of Large-Capacity Lithium-Ion Battery and Evaluation of Battery System for Telecommunications. Journal of Power Sources, 244, 11-16. https://doi.org/10.1016/j.jpowsour.2013.01.155

[2] Tang, X.P., Zou, C.F., Yao, K., Chen, G.H., Liu, B.Y., He, Z.W. and Gao, F.R. (2018) A Fast Estimation Algorithm for Lithium-Ion Battery State of Health. Journal of Power Sources, 396, 453-458. https://doi.org/10.1016/j.jpowsour.2018.06.036

[3] Madhuri Bayya, Dr. BVSSN. Prabhakara, U.M. Rao, Dr.N.Moorthy(2017)Battery State Estimation using AC analysis 5th Edition, Energy Procedia 117 (2017) 739-744. https://doi.org/10.1016/j.egypro.2017.05.189

[4] Wang, X.J. and Fan, L.X. (2012) The Application Research of MD5 Encryption Algorithm in DCT Digital Watermarking. Physics Procedia, 25, 1264-1269. https://doi.org/10.1016/j.phpro.2012.03.231

[5] Min, H. (1998) Blockchain Technology for Enhancing Supply Chain Resilience. BUSHOR-1529; No. of Pages 11.

[6] Reyna, A., Martín, C., Chen, J., Soler, E. and Díaz, M. (2017) On Blockchain and Its Integration with IoT. Future Generation Computer Systems, 88, 173-190. https://doi.org/10.1016/j.future.2018.05.046

[7] Kong, X.R., Bonakdarpour, A., Wetton, B.T., Wilkinson, D.P. and Gopaluni, B. (2018) State of Health Estimation for Lithium-Ion Batteries. IFAC PapersOnLine, 51-18, 667-671.

[8] Bunder, M., Nitaj, A., Susilo, W. and Tonien, J. (2017) A Generalized Attack on RSA Type Cryptosystems. Theoretical Computer Science, 704, 74-81. https://doi.org/10.1016/j.tcs.2017.09.009 